\title{
Exchange interactions in random magnetic overlayers
}

\author{
J. Kudrnovský ${ }^{\mathrm{a}, *}$, V. Drchal ${ }^{\mathrm{a}}$, I. Turek ${ }^{\mathrm{b}, \mathrm{c}}$, P. Bruno ${ }^{\mathrm{d}}$ \\ a Institute of Physics, Academy of Sciences of the Czech Republic, Na Slovance 2, CZ-182 21 Praha 8, Czech Republic \\ ${ }^{\mathrm{b}}$ Institute of Physics of Materials, Academy of Sciences of the Czech Republic, Žižkova 22, CZ-616 62 Brno, Czech Republic \\ ${ }^{\mathrm{c}}$ Department of Electronic Structures, Charles University, Ke Karlovu 5, CZ-121 16 Prague, Czech Republic \\ ${ }^{\mathrm{d}}$ Max-Planck-Institut für Mikrostrukturphysik, Weinberg 2, D-06120 Halle, Germany
}

\begin{abstract}
We have determined the effective exchange interactions in the random magnetic overlayers on a nonmagnetic substrate by a mapping of the accurate ab initio total energies onto a classical two-dimensional Heisenberg Hamiltonian. The electronic structure is determined within the tight-binding linear muffin-tin orbital method combined with the surface Green function technique while the effect of a disorder is included using the coherent potential approximation. We employ both the magnetic-force and the vertex-cancellation theorems to evaluate the concentration dependence on the overlayer exchange interactions. The formalism is applied to the magnetic overlayers on the fcc$\mathrm{Cu}(0001)$ substrate. (C) 2002 Elsevier Science B.V. All rights reserved.
\end{abstract}

Keywords: Density functional calculations; Green's function methods; Alloys; Magnetic films

\section{Introduction}

Exchange interactions between the magnetic atoms in the non-magnetic as well as in ferromagnetic metals and their alloys are of a key importance for understanding of the nature of magnetism in such systems. These interactions, determined from the first principles, play also an important role in the construction of the model magnetic Hamiltonians, e.g., the classical Heisenberg Hamiltonian, which allows to understand the temperature dependent properties of the complex magnetic systems [1] as well as their dynamic properties, like for example spin-wave spectra.

\footnotetext{
${ }^{*}$ Corresponding author. Tel.: +420-2-858-8605; fax: +420-2868-90527.

E-mail address: kudrnov@fzu.cz (J. Kudrnovský).
}

Such a study can be also extended to two-dimensional layered systems as it was recently demonstrated [2]. While a reasonable progress has been achieved in understanding of the spin-wave spectra and thermodynamic properties like the Curie temperature of the elemental ferromagnets [1] and, recently, also of the ideal layered systems [2], there is a small progress as concerns on evaluation of the related properties in the magnetic substitutional alloys [3]. This is particularly true for systems with surfaces and interfaces playing a key role in various technological applications. It is thus important to have a theory as well as the first-principle computational scheme suitable for evaluation of the exchange interactions in the random substitutional alloys, their surfaces and interfaces. This would allow to understand their properties on a level comparable to ideal (bulk) crystals. We have recently developed such a scheme for bulk alloys 
[4] and a purpose of this paper is to extend this study to random two-dimensional systems.

\section{Formalism}

The exchange interactions for random alloys are conveniently evaluated in the framework of the real-space approach [3] which is applicable also to systems with broken translational symmetry in contrast to the conventional reciprocal-spacebased approaches, such as the frozen-magnon approach [5]. Both approaches adopt the adiabatic approximation in which the calculated exchange energies are small compared to the bandwidth and the exchange splitting of each magnetic atom in a system. As we are interested in the exchange interactions among different sites in the system, we can neglect the intracell non-collinearity. We employ the magnetic force theorem $[3,6]$ according to which the change of the total energy of a system coincides with the sum of one-particle energy changes of occupied states. Another important point is the vertex-cancellation theorem (VCT) valid within the framework of the coherent-potential approximation (CPA). The VCT can be used to calculate any kind of the energy associated with a local rotation of the magnetization, including the Heisenberg exchange interactions which are of interest here. This theorem states that disorder-induced vertex corrections can be neglected in the limit of infinitesimal rotations of magnetizations and it represents a good approximation for the case of small but finite rotations [7].

The effective two-dimensional alloy Heisenberg Hamiltonian with classical spins is

$H^{\mathrm{eff}}=-\sum_{i \neq j} J_{i j} \mathbf{e}_{i} \cdot \mathbf{e}_{j}$,

where $J_{i j}$ is the exchange interaction energy between two particular sites $i, j$ in a random alloy, and $\mathbf{e}_{i}, \mathbf{e}_{j}$ are the unit vectors pointing in the direction of local magnetic moments at sites $i, j$, respectively. The quantities $J_{i j}$ assume four different values in a random overlayer depending on the occupation of terminal sites $i, j$, namely $J_{i j}^{Q, Q^{\prime}}$, $Q, Q^{\prime}=A, B$. In the framework of the ab initio tight-binding linear muffin-tin orbital method (TBLMTO) [8], $J_{i j}^{Q, Q^{\prime}}$ are given as [4]
$J_{i j}^{Q, Q^{\prime}}=\frac{1}{4 \pi} \operatorname{Im} \int_{C} \operatorname{tr}_{L}\left\{\Delta_{i}^{Q}(z) \bar{g}_{i j}^{Q, Q^{\prime}, \uparrow}(z) \Delta_{j}^{Q^{\prime}}(z) \bar{g}_{j i}^{Q^{\prime}, Q, \downarrow}(z)\right\} \mathrm{d} z$,

$\Delta_{i}^{Q}(z)=P_{i}^{Q, \uparrow}(z)-P_{i}^{Q, \downarrow}(z)$.

In this expression, $\operatorname{tr}_{L}$ denotes the trace over the angular momentum $L=(\ell m)$, the energy integration is performed in the upper half of the complex energy plane over a contour $C$ starting below the bottom of the valence band and ending at the Fermi energy, while $P_{i}^{Q, \sigma}(z)$ are diagonal matrices of the potential functions of the TB-LMTO method for a given atom type and spin orientation $\sigma=\uparrow, \downarrow$ with elements $P_{i, L}^{\sigma}(z)$, and $\Delta_{i}^{Q}(z)$ plays a role of the local exchange splitting for an atom $Q$ in the conventional tight-binding approach. The sites $i, j$ are limited to the random overlayer, i.e., we neglect very small magnetic moments, induced by the magnetic overlayer in a non-magnetic semi-infinite substrate. The interaction of two spins is mediated by the conditionally averaged (auxiliary) Green functions $\bar{g}_{i j}^{Q, Q^{\prime}, \uparrow}(z)$ and $\bar{g}_{j i}^{Q^{\prime}, Q, \downarrow}(z)$. In the framework of the CPA, they are evaluated as [8]

$$
\begin{aligned}
& \bar{g}_{i j}^{Q, Q^{\prime}, \sigma}(z)=\tilde{f}_{i}^{Q, \sigma}(z) \bar{g}_{i j}^{\sigma}(z) f_{j}^{Q^{\prime}, \sigma}(z), \\
& f_{i}^{Q, \sigma}(z)=\left[1+\left(P_{i}^{Q, \sigma}(z)-\mathscr{P}_{i}^{\sigma}(z)\right) \bar{g}_{i i}^{\sigma}(z)\right]^{-1}, \\
& \tilde{f}_{i}^{Q, \sigma}(z)=\left[1+\bar{g}_{i i}^{\sigma}(z)\left(P_{i}^{Q, \sigma}(z)-\mathscr{P}_{i}^{\sigma}(z)\right)\right]^{-1} .
\end{aligned}
$$

The coherent potential function $\mathscr{P}_{i}^{\sigma}(z)$ is the sitediagonal matrix with the elements $\mathscr{P}_{i, L L^{\prime}}^{\sigma}(z), \bar{g}_{i i}^{\sigma}(z)$ and $\bar{g}_{i j}^{\sigma}(z)$ are the site-diagonal and the site off-diagonal blocks of the configurationally averaged auxiliary Green function [8], respectively, evaluated with the help of the lattice Fourier transform (for more details see [8]). The Fourier transform of $J_{i j}^{Q, Q^{\prime}}$ can be calculated in two ways, namely by a summation over the large number of the coordination shells

$J^{Q, Q^{\prime}}\left(\mathbf{q}_{\|}, z\right)=\sum_{j(j \neq 0)} J_{0 j}^{Q, Q^{\prime}}(z) \mathrm{e}^{i \mathbf{q}_{\|} \cdot \mathbf{R}_{j}}$

or, by a direct Fourier transformation of Eq. (2), namely

$$
\begin{aligned}
J^{Q, Q^{\prime}}\left(\mathbf{q}_{\|}, z\right)= & \frac{1}{4 \pi N_{\|}} \operatorname{Im} \sum_{\mathbf{k}_{\|}} \int_{C} \operatorname{tr}_{L}\left\{\Delta^{Q}(z) \bar{g}^{Q, Q^{\prime}, \uparrow}\left(\mathbf{k}_{\|}, z\right)\right. \\
& \left.\times \Delta^{Q^{\prime}}(z) \bar{g}^{Q^{\prime}, Q, \downarrow}\left(\mathbf{k}_{\|}-\mathbf{q}_{\|}, z\right)\right\} \mathrm{d} z .
\end{aligned}
$$


Here, the sum runs over the corresponding surface Brilouin zone (SBZ), and $N_{\|}$is the number of $\mathbf{k}_{\|^{-}}$ points in the SBZ. It should be noted that the quantity $J_{i j}^{Q, Q^{\prime}}$ is closely related to the configurationally averaged non-interacting static transversal susceptibility of a random overlayer.

The derivation of the above expressions is quite similar to that for bulk alloys [4]. One should, however, mention that Eq. (2) can be derived in two ways: (i) by performing the rotation of magnetizations at two sites $i$ and $j$ occupied by atoms $Q$ and $Q^{\prime}$, respectively, for a given alloy configuration, and then applying the CPA configurational average, or, (ii) by rotating the magnetizations of two sites $i$ and $j$ occupied by the 'effective' CPA atoms, i.e., using the same approach as for nonrandom systems [2] but applied to the configurationally averaged system. The equivalence of both approaches is obvious from a physical point of view (the interchange of a configurational average and of a small rotation of magnetizations at two sites) but it is non-trivial from a formal point of view. In both cases, one has to employ the VCT to derive Eq. (2).

\section{Results and discussion}

It should be noted that two-dimensional Heisenberg Hamiltonian (1) is obtained by a mapping the total energies of a realistic system, consisting of a random magnetic overlayer sandwiching the semi-infinite non-magnetic substrate and the semiinfinite vacuum described in terms of empty spheres
[8]. In this way direct interactions among magnetic atoms in the overlayer as well as the indirect interactions of magnetic atoms via the substrate are included into the construction of the Heisenberg model. Such indirect interactions can play an important role in the explanation of some phenomena in magnetic multilayers (see [2] for more details).

The potential functions and Green functions, appearing in Eq. (2), were determined within the non-relativistic TB-LMTO-CPA method [8] assuming the experimental $\mathrm{Cu}$ lattice constant and the exchange potential in the form suggested by Vosko-Wilk-Nusair [9]. The Gaussian quadrature method with 20 nodes was used to perform the energy integration on the semi-circle $C$ in the upper part of the complex energy plane. The integration over the full SBZ was performed using up to $150000 \mathbf{k}_{\|}$-vectors for the energy point on the contour $C$ closest to the Fermi energy, and then the number of $\mathbf{k}_{\|}$-vectors progressively decreasing for more distant energy points, and for points close to the bottom of the band. The exchange interactions were calculated up to 48th shell.

We have performed calculations for the reference systems $\mathrm{Fe} / \mathrm{Cu}\left(\begin{array}{lll}0 & 0 & 1\end{array}\right)$ and $\mathrm{Co} / \mathrm{Cu}\left(\begin{array}{lll}0 & 0 & 1\end{array}\right)$ and for their disordered counterparts, namely $\mathrm{Fe}_{50} \mathrm{Co}_{50} /$

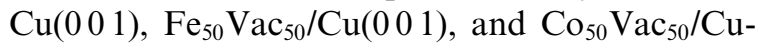
(0 01 1). Here Vac denotes vacancies (empty sites) and the corresponding models represent incomplete overlayers of magnetic atoms on the fcc$\mathrm{Cu}\left(0 \begin{array}{ll}0 & 1)\end{array}\right)$ substrate. In each case, the overlayers are 1 ML thick.

The results are summarized in Table 1 and Figs. 1-3. The local moments on Fe-sites are enhanced

Table 1

Calculated values of the local Fe- and Co-magnetic moments in the overlayer and the first nearest-neighbor exchange interactions among $\mathrm{Fe}-\mathrm{Fe}, \mathrm{Fe}-\mathrm{Co}$, and $\mathrm{Co}-\mathrm{Co}$ pairs in various overlayers

\begin{tabular}{llllll}
\hline overlayer & $\mathrm{Fe}$ & $\mathrm{Fe}_{50} \mathrm{Vac}_{50}$ & $\mathrm{Fe}_{50} \mathrm{Co}_{50}$ & $\mathrm{Co}_{50} \mathrm{Vac}_{50}$ & $\mathrm{Co}$ \\
\hline$m^{\mathrm{Fe}}\left(\mu_{B}\right)$ & 2.76 & 2.68 & 2.81 & - & 1.60 \\
$m^{\mathrm{Co}}\left(\mu_{B}\right)$ & 1.69 & - & 1.73 & - & 2.87 \\
$J_{1}^{\mathrm{Fe}, \mathrm{Fe}}(\mathrm{mRy})$ & 2.82 & 4.69 & 2.87 & - & 3.77 \\
$J_{1}^{\mathrm{Fe}, \mathrm{Co}}(\mathrm{mRy})$ & 2.69 & - & 2.78 & 3.31 & 2.84 \\
$J_{1}^{\mathrm{Co}, \mathrm{Co}}(\mathrm{mRy})$ & 2.10 & - & 2.23 & 2.32 \\
\hline
\end{tabular}

In the case of the ideal $\mathrm{Fe} / \mathrm{Cu}(001)$ overlayer the $\mathrm{Co}-\mathrm{Co}$ term corresponds to two isolated Co impurities in the $\mathrm{Fe}$ layer while the $\mathrm{Fe}-$ $\mathrm{Co}$ term describes a single $\mathrm{Co}$ impurity in the Fe layer, and similarly for the $\mathrm{Co} / \mathrm{Cu}(001)$ overlayer. Exchange interactions among the magnetic atoms and vacancies (or among two vacancies) are negligible, similarly as the local magnetic moments on vacancies and therefore are not shown. 


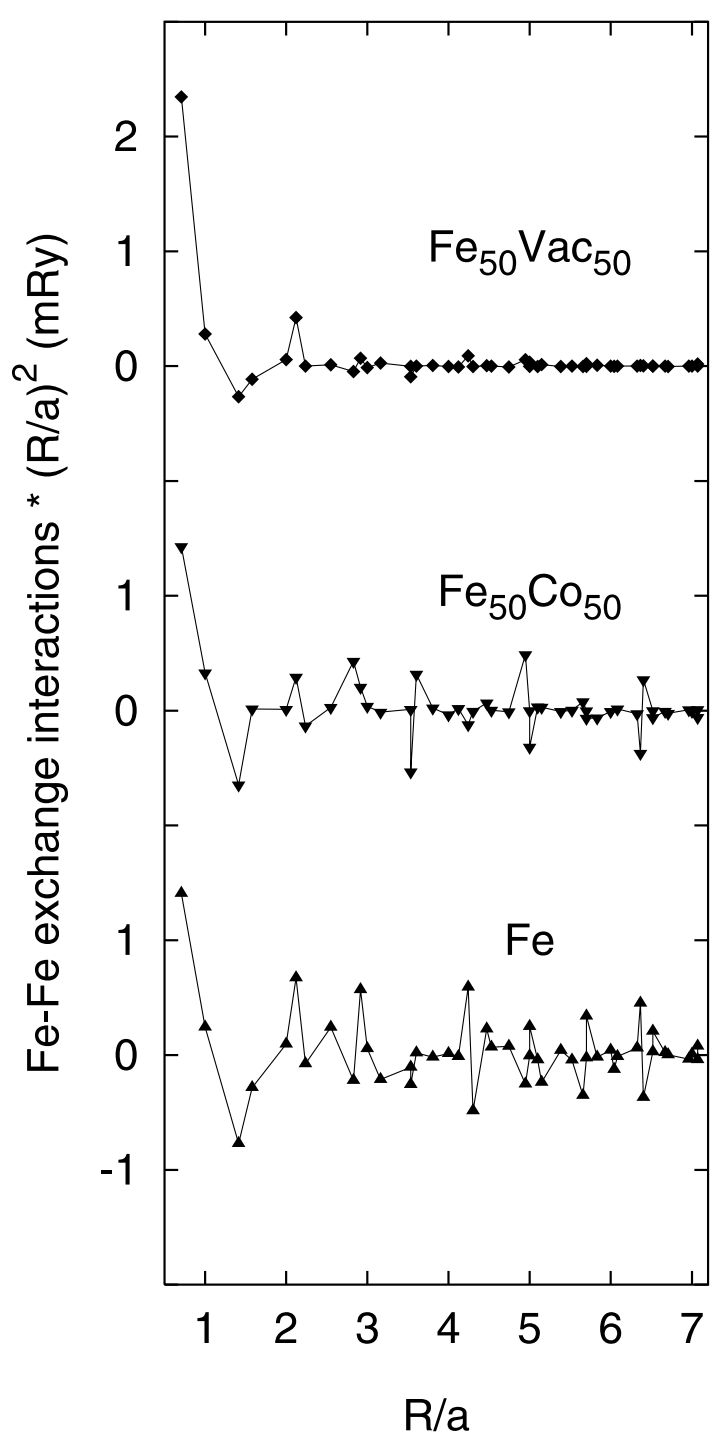

Fig. 1. Effective pair interactions among the $\mathrm{Fe}-\mathrm{Fe}$ pairs in the magnetic overlayers on the non-magnetic fcc- $\mathrm{Cu}\left(\begin{array}{ll}0 & 0\end{array}\right)$ substrate as a function of their distance $R$ (in units of the lattice constant a): the $\mathrm{Fe}_{50} \mathrm{Vac}_{50}$ overlayer (top panel), the $\mathrm{Fe}_{50} \mathrm{Co}_{50}$ overlayer (middle panel), and the $\mathrm{Fe}$ overlayer (bottom panel). Note that the pair interactions are multiplied by a factor $(R / a)^{2}$.

compared to the bulk bcc-Fe crystal due to the reduced coordination of atoms in the overlayer. On contrary, the local moments on Co-sites are only slightly different from the bulk fcc value because the majority band is nearly filled and thus varying environment does not change the occu-

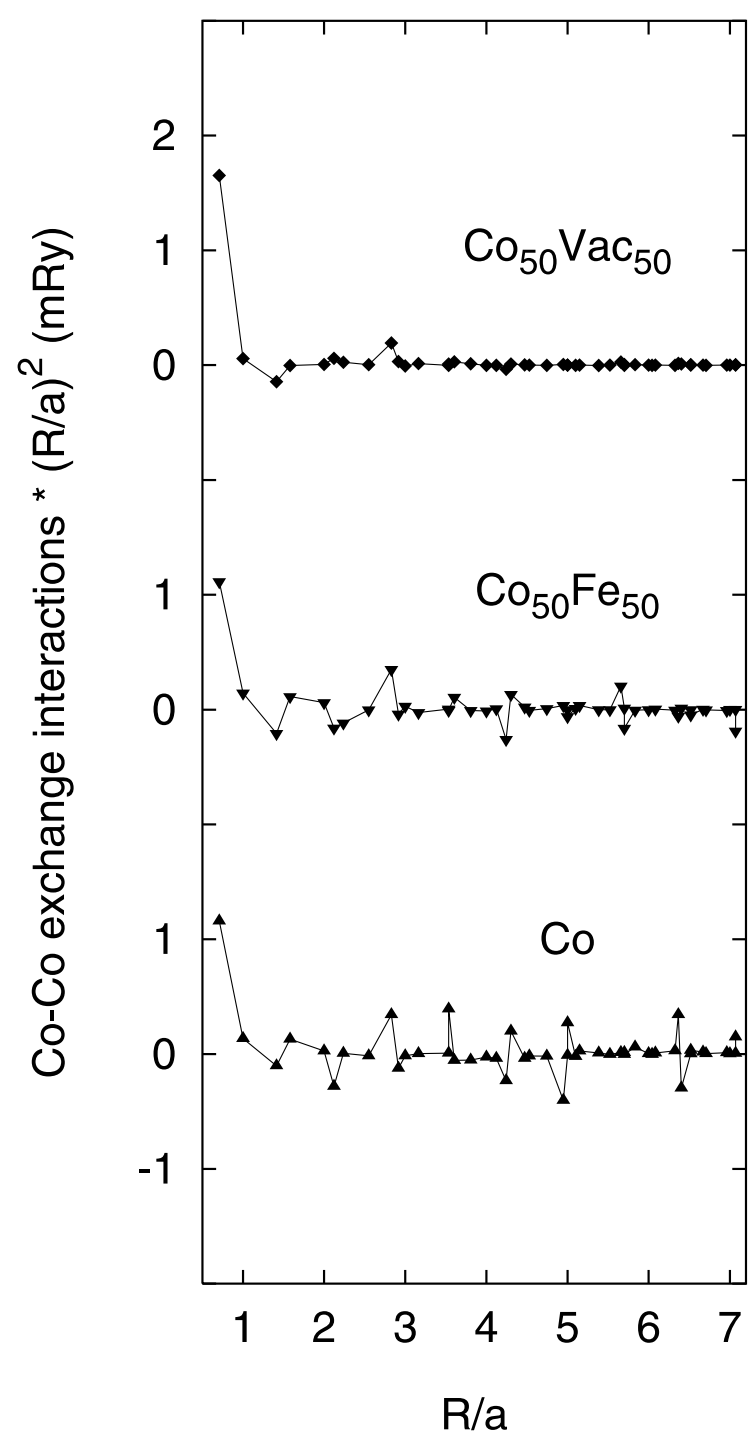

Fig. 2. Effective pair interactions among the $\mathrm{Co}-\mathrm{Co}$ pairs in the magnetic overlayers on the non-magnetic fcc- $\mathrm{Cu}\left(\begin{array}{lll}0 & 0 & 1\end{array}\right)$ substrate as a function of their distance $R$ (in units of the lattice constant a): the $\mathrm{Co}_{50} \mathrm{Vac}_{50}$ overlayer (top panel), the $\mathrm{Co}_{50} \mathrm{Fe}_{50}$ overlayer (middle panel), and the Co overlayer (bottom panel). Note that the pair interactions are multiplied by a factor $(R / a)^{2}$.

pation of spin subbands so strongly as in the case of the bulk $\mathrm{Fe}$ with a partially filled majority band (see [10] for more details). This is due to a strong ferromagnetism which is stabilized for the $\mathrm{Fe}_{1-x} \mathrm{Co}_{x}$ overlayer $(0 \leqslant x \leqslant 1)$ and leads to above mentioned enhancement of $\mathrm{Fe}$ local magnetic 


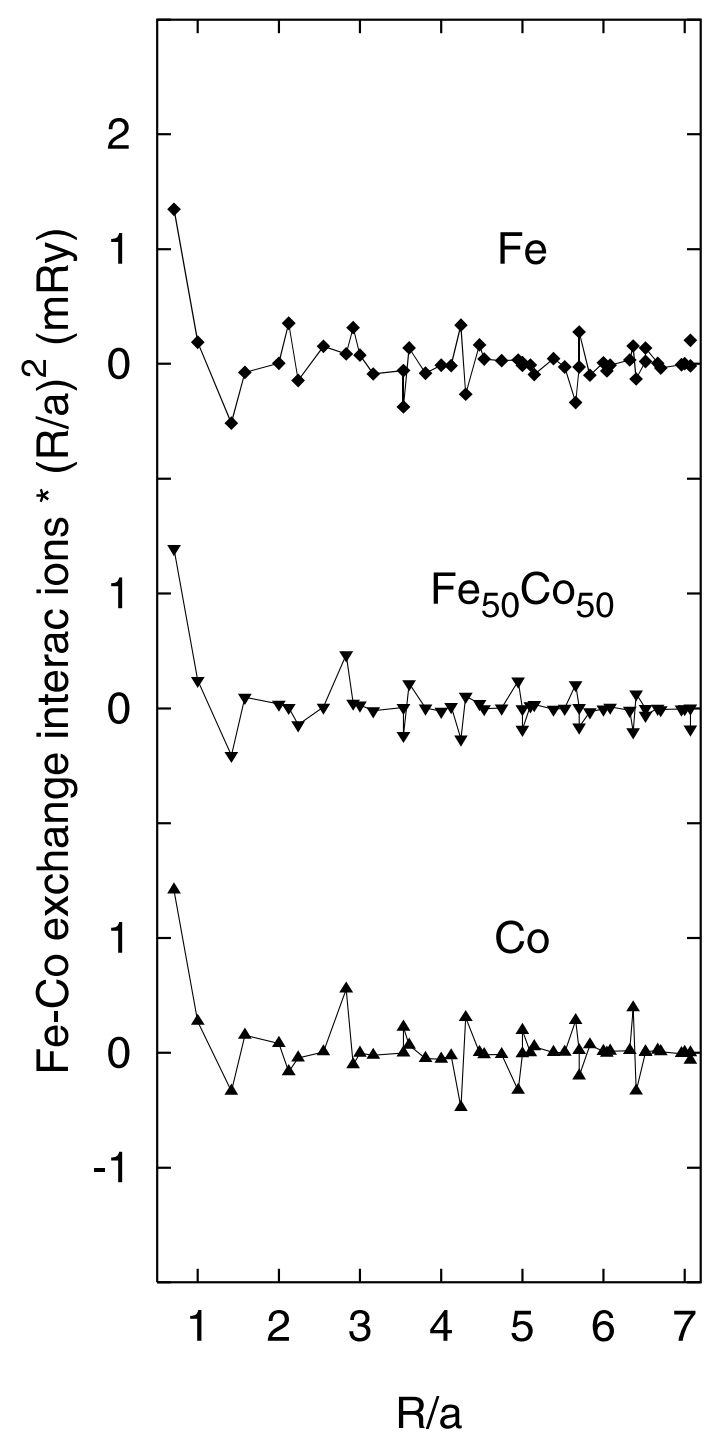

Fig. 3. Effective pair interactions among the $\mathrm{Fe}-\mathrm{Co}$ pairs in the magnetic overlayers on the non-magnetic fcc- $\mathrm{Cu}\left(\begin{array}{ll}0 & 0\end{array}\right)$ substrate as a function of their distance $R$ (in units of the lattice constant $a$ ): the Fe overlayer (top panel), the $\mathrm{Fe}_{50} \mathrm{Co}_{50}$ overlayer (middle panel), and the Co overlayer (bottom panel). In the case of the $\mathrm{Fe}$ overlayer the pair interactions correspond to a single Coimpurity case, and similarly for the Co overlayer. Note that the pair interactions are multiplied by a factor $(R / a)^{2}$.

moments as well as to a weak concentration dependence of both the local $\mathrm{Fe}$ and Co magnetic moments [10].The majority spin electrons are only weakly influenced by the disorder in the $\mathrm{Fe}_{50} \mathrm{Co}_{50}$ overlayer so that this subband exhibits nearly a rigid-band behavior. On contrary, the minority electrons are influenced by a disorder more strongly. The present results are also in a fairly good agreement with full-potential slab calculations for ideal overlayers [11]. The disorder is, however, very strong in the case of a partial monolayer coverage of fcc- $\mathrm{Cu}(001)$ substrate by the magnetic $\mathrm{Fe}$ and $\mathrm{Co}$ atoms due to a large difference between the d-levels of magnetic atoms and vacancies (the split-band regime of the CPA). In this case, the rigid-band model is not applicable. Also these overlayers exhibit a strong ferromagnetism, i.e., the filled majority d-subband, and a small concentration variation of local magnetic moments with a coverage. The magnetic polarization of empty sites is (negligible being less than $\left.0.01 \mu_{B}\right)$.

The leading values of the pair exchange interactions for ideal overlayers, namely $J_{1}^{\mathrm{Fe}, \mathrm{Fe}}$ for $\mathrm{Fe} /$ $\mathrm{Cu}\left(\begin{array}{lll}0 & 0 & 1\end{array}\right)$ and $J_{1}^{\mathrm{Co}, \mathrm{Co}}$ for $\mathrm{Co} / \mathrm{Cu}\left(\begin{array}{lll}0 & 0 & 1\end{array}\right)$, agree with previous calculations [2]; $J_{1}^{\mathrm{Fe}, \mathrm{Fe}}$ are essentially the same as in $\mathrm{Fe} / \mathrm{Cu}(001), \mathrm{Fe}_{50} \mathrm{Co}_{50} / \mathrm{Cu}(001)$, as well as for the two impurity case in $\mathrm{Co} / \mathrm{Cu}(001)$ overlayer. A similar behavior is also found for $J_{1}^{\mathrm{Co}, \mathrm{Co}}$ in $\mathrm{Co} / \mathrm{Cu}\left(\begin{array}{lll}0 & 0 & 1\end{array}\right), \mathrm{Fe}_{50} \mathrm{Co}_{50} / \mathrm{Cu}\left(\begin{array}{lll}0 & 0 & 1\end{array}\right)$, and $\mathrm{Fe} / \mathrm{Cu}\left(\begin{array}{lll}0 & 0 & 1\end{array}\right)$ overlayers. On contrary, both $J_{1}^{\mathrm{Fe}, \mathrm{Fe}}$ and $J_{1}^{\mathrm{Co}, \mathrm{Co}}$ are strongly enhanced in $\mathrm{Fe}_{50} \mathrm{Vac}_{50} / \mathrm{Cu}\left(\begin{array}{lll}0 & 0 & 1)\end{array}\right)$ and $\mathrm{Co}_{50} \mathrm{Vac}_{50} / \mathrm{Cu}(001)$ due to a strongly reduced coordination of magnetic atoms in these overlayers. A similar trend of increasing $J_{1} \mathrm{~s}$ with the reduced coordination of magnetic atoms, although not so pronounced, was found for magnetic monolayers embedded in the host fcc-Cu crystal, deposited on fcc- $\mathrm{Cu}(001)$ as overlayers, and for the free standing magnetic layers [2].

The dependence of the exchange pair interactions $J^{\mathrm{Fe}, \mathrm{Fe}}(R), J^{\mathrm{Co}, \mathrm{Co}}(R)$, and $J^{\mathrm{Fe}, \mathrm{Co}}(R)$ on the distance $R=\left|\mathbf{R}_{i}-\mathbf{R}_{j}\right|$ is presented in Figs. 1-3, respectively. To be more precise, the dependence $R^{2} J^{Q, Q^{\prime}}(R)$, where $Q, Q^{\prime}=\mathrm{Fe}$, Co is shown there. In the limit of large values of $R$, the expression (2) can be evaluated analytically by means of the stationary phase approximation [12]. For the bulk, one obtains $J^{Q, Q^{\prime}}(R) \propto R^{-3}$ [13], while a similar estimate gives $J^{Q, Q^{\prime}}(R) \propto R^{-2}$ for a two-dimensional case. It should be noted, however, that the present case is not strictly two-dimensional due to the indirect exchange interactions of two magnetic 
atoms in the overlayer via a non-magnetic fcc- $\mathrm{Cu}\left(\begin{array}{ll}0 & 0\end{array}\right)$ substrate. This is also seen in Figs. 13 for the ideal overlayers: the $R^{-2}$ dependence, clearly dominates but an overall behavior is more complex. In a disordered system one expects an additional damping due to an exponential decay of the configurationally averaged Green functions evaluated in the CPA, $\bar{g}_{i j}^{\sigma}(z)$, with the distance $R$. In the case of a weak disorder in one spin channel (majority spins in $\mathrm{Fe}_{50} \mathrm{Co}_{50} / \mathrm{Cu}(001)$ ), we observe only a weak additional damping in contrast to the strong split-band type of disorder in both spin channels for the case of the incomplete magnetic overlayers.

In conclusion, a construction of the effective two-dimensional alloy Heisenberg Hamiltonian represents an important, but only the very first step in studying the relevant properties of an overlayer, such as the magnon dispersion laws, the spin-wave stiffness, and the evaluation of the Curie temperature. This will represent a natural generalization of the approach already existing for ideal magnetic overlayers [2].

\section{Acknowledgements}

J.K., I.T., and V.D. acknowledge the financial support provided by the Grant Agency of the Czech Republic (No. 202/00/0122), the Grant Agency of the Academy of Sciences of the Czech Republic (No. A1010829), the Czech Ministry of
Education, Youth and Sports (COST P3.40 and P3.70), the CMS Vienna (GZ 45.504), and the RTN 'Computational Magnetoelectronics' (HPRN-CT-2000-00143).

\section{References}

[1] V.P. Antropov, B.N. Harmon, A.N. Smirnov, J. Magn. Magn. Mater. 200 (1999) 148.

[2] M. Pajda, J. Kudrnovský, I. Turek, V. Drchal, P. Bruno, Phys. Rev. Lett. 85 (2000) 5424.

[3] A.I. Liechtenstein, M.I. Katsnelson, V.P. Antropov, V.A. Gubanov, J. Magn. Magn. Mater. 67 (1987) 65.

[4] P. Bruno, J. Kudrnovský, unpublished.

[5] S.V. Halilov, H. Eschrig, A.Y. Perlov, P.M. Oppeneer, Phys. Rev. B 58 (1998) 293.

[6] A. Oswald, R. Zeller, P.J. Braspenning, P.H. Dederichs, J. Phys. F: Met. Phys. 15 (1985) 193.

[7] P. Bruno, J. Kudrnovský, V. Drchal, I. Turek, Phys. Rev. Lett. 76 (1996) 4254;

J. Kudrnovský, V. Drchal, I. Turek, P. Bruno, P. Dederichs, P. Weinberger, in: H. Dreyssé (Ed.), Electronic Structure and Physical Properties of Solids, Lecture Notes in Physics, 535, Springer, Berlin, 2000, p. 313.

[8] I. Turek, V. Drchal, J. Kudrnovský, M. Šob, P. Weinberger, Electronic Structure of Disordered Alloys, Surfaces and Interfaces, Kluwer, Boston-London-Dordrecht, 1997.

[9] S.H. Vosko, L. Wilk, M. Nusair, Can. J. Phys. 58 (1980) 1200.

[10] I. Turek, J. Kudrnovský, V. Drchal, P. Weinberger, Phys. Rev. B 49 (1994) 3352.

[11] C. Li, A.J. Freeman, C.L. Fu, J. Magn. Magn. Mater. 83 (1990) 51.

[12] P. Bruno, Phys. Rev. B 52 (1995) 411.

[13] M. Pajda, J. Kudrnovský, I. Turek, V. Drchal, P. Bruno, Phys. Rev. B 64 (2001) 174402. 